\title{
Basal to Non-Basal Transition for In-Plane Deformation of AZ31 Magnesium Alloys
}

\author{
J. BALÍK ${ }^{a, *}$, P. LUKÁC̆ ${ }^{a}$ AND R. KUŽEL ${ }^{b}$
}

${ }^{a}$ Department of Physics of Materials, Charles University, MFF, Ke Karlovu 5, 12116 Prague 2, Czech Republic ${ }^{b}$ Department of Condensed Matter Physics, Charles University, MFF, Ke Karlovu 5, 12116 Prague 2, Czech Republic

The increases in strain hardening observed for the in-plane tensile deformation of well-aged magnesium alloy AZ31 are related to a transition from basal to non-basal slip. Based on the results of texture measurements, the double prismatic slip is proposed as the dominant secondary mechanism. The necessary fast strengthening of the basal slip is modelled by an accommodation contribution to the grain boundary resistance. The transition may be consistently modelled by involving the accommodation rather than the production of new sessile dislocations into the evolution laws for dislocation populations.

PACS: $62.20 . \mathrm{F}-$, 81.40.Ef

\section{Introduction}

Rolled sheets of the Mg-based alloys such as AZ31, which are of a great practical significance, have strong basal texture with a characteristic splitting of c-poles maxima towards the rolling direction from the normal direction $[1,2]$. As reported in the literature [1, 3], tensile in-plane deformation of such samples should occur practically with near-zero tensile twinning. In an earlier paper [4], rather sharp initial increases (lifts) of strain hardening coefficient were revealed for in-plane tension of AZ31 sheets and well-aged conditions. In the usual stress-strain curves, these lifts correspond to short S-shaped periods $\leq 1 \%$ of the strain followed by a parabolic part characteristic of a continuous slip mode. Because tensile twinning is nominally excluded by the respective conditions, the appearance of the lifts has not been related to the twinning but to the transition from the initial basal slip to the next non-basal one.

The aim of the present paper is to extend the analysis of early deformation stages of rolled sheets of AZ31 that was introduced in [4]. Further tensile tests and measurements of the texture after deformation were conducted. An evolution model for the primary and secondary dislocation densities is used to describe the phenomenon of hardening lifts including the anisotropy with respect to the original rolling direction.

\section{Experimental results 2.1. Tensile tests}

The rolled sheet of the alloy AZ31 (3 wt\% Al, $1 \mathrm{wt} \% \mathrm{Zn}$, balance $\mathrm{Mg}$ ) was in-plane tensile tested in the directions parallel (RD) and perpendicular (TD) to the rolling directions at a strain rate of $1.3 \times 10^{-3} \mathrm{~s}^{-1}$ and at temperatures $23,100,150$ and $200^{\circ} \mathrm{C}$. Besides the as-received state H24 with grain sizes from 6 to $40 \mu \mathrm{m}$ and significant fraction of twins, the state aged at $300^{\circ} \mathrm{C}$ for $8 \mathrm{~h}$

\footnotetext{
* corresponding author; e-mail: balik@svum.cz
}

with equiaxed grain size of $15 \mu \mathrm{m}$ and practically free of twins was examined. For microstructure details see [5], where also the tensile curves have been published. Here we deal with the Mecking plots, i.e., the strain hardening coefficient versus the flow stress, the examples of which for two selected temperatures are given in Fig. 1a, b. A qualitative difference in the form of initial parts of the Mecking plots is observed at temperatures up to $150^{\circ} \mathrm{C}$. Whereas the $300 / 8$ structure reveals the hardening lifts mentioned in introduction that were related to the transition between the primary to secondary glide mechanisms, the H24 state exhibits much greater hardening values that, however, decrease continuously over the whole stress range. This hardening excess in the H24 state was connected [4] to the gradual plastification of grains due to the existence of predeformations, i.e., higher yield points in individual grains stemming from the cold predeformation history.

\subsection{Textures}

In the initial state, the typical basal texture is observed with the characteristic spread of basal fibres towards the rolling direction is observed, Fig. 2a. After in-plane deformation, the gathering of the prismatic poles (10 $\overline{1} 0)$ towards the tensile directions irrespective of the initial texture seems to be a general feature, for an example see Fig. 2b. For this behaviour to analyze, we will assume that, in each grain, the activity of a single slip prevails, governed by the Schmid law. These assumptions were repeatedly employed successfully and confirmed experimentally for in-plane deformation, see [6].

By means of the Schmid analysis, both the basal and pyramidal slip have to be excluded as the overwhelming mechanisms because the load axis would tend to $\langle a\rangle-$ and $\langle c\rangle$-directions, respectively. On the other hand, the activity of the prismatic slip $\{10 \overline{1} 0\}\langle\overline{1} 2 \overline{1} 0\rangle$ should move the load direction always towards the double prismatic position, see Fig. 3. Then, under the condition of double prismatic slip, the observed limit $\langle 10 \overline{1} 0\rangle$ is approached. 

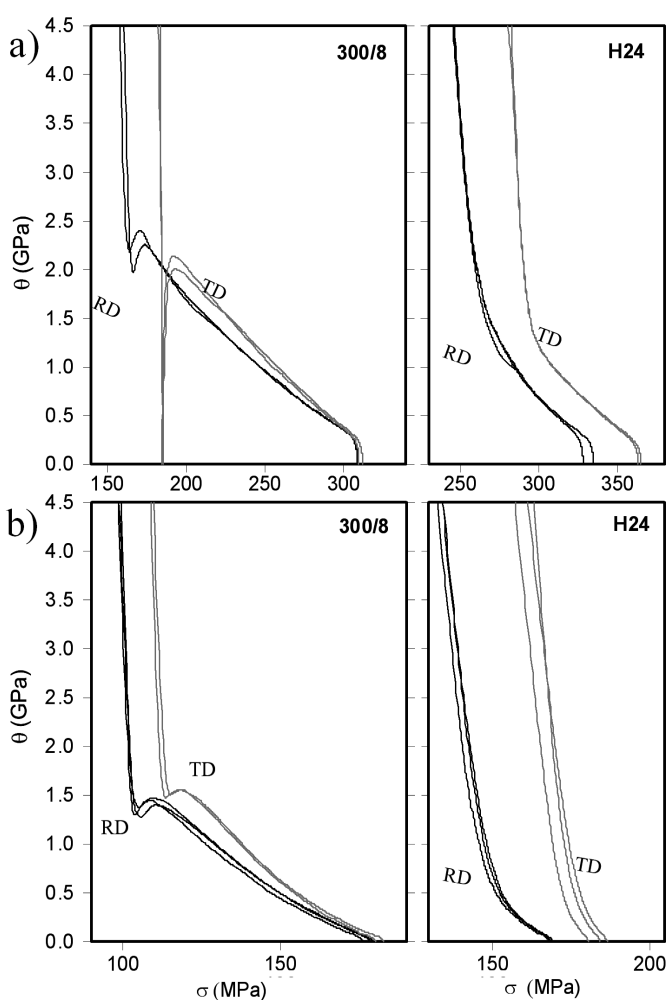

Fig. 1. Tensile deformation of the sheets AZ31: (a) $23{ }^{\circ} \mathrm{C}$, (b) $150{ }^{\circ} \mathrm{C}$.

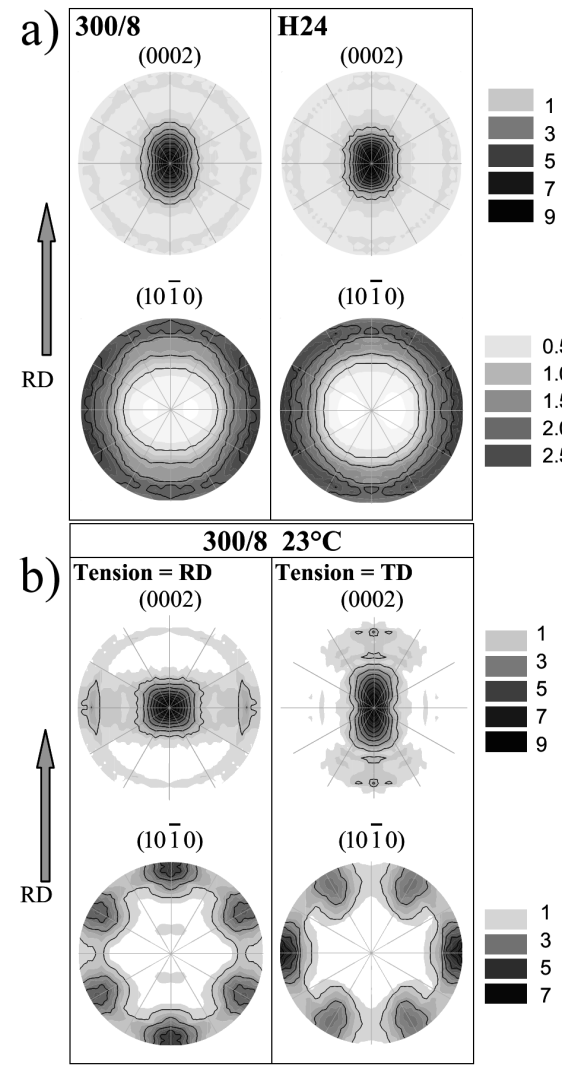

Fig. 2. (a) Initial states of the AZ31 sheet; (b) sheet AZ31, state $300 / 8$, failed in tension at $23^{\circ} \mathrm{C}$.

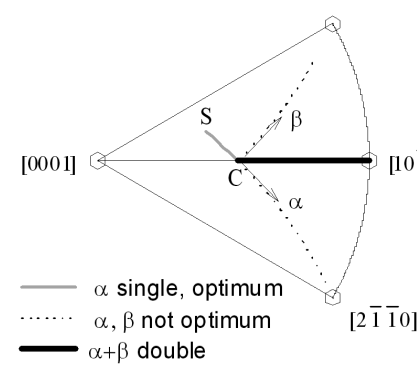

Prismatic Schmid factors

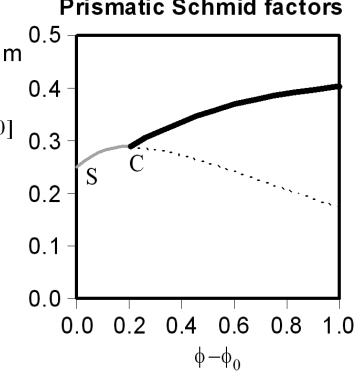

Fig. 3. Operation of adjacent prismatic slip systems. $\alpha=(01 \overline{1} 0)[2 \overline{1} \overline{1} 0], \beta=(1 \overline{2} 10)[11 \overline{2} 0]$. The trajectory shown starts at $\mathrm{S}$. The prismatic Schmid factors are plotted versus the true strain.

\section{Discussion}

\subsection{Hardening due to accommodation}

In the present work, we attempt to model the primary/ secondary transition without considering any activity of $\langle c+a\rangle$ dislocations which led in our former paper [4] to the creation of extra strong obstacles. Such an alternate model might be suitable for the case of secondary prismatic slip as reported above.

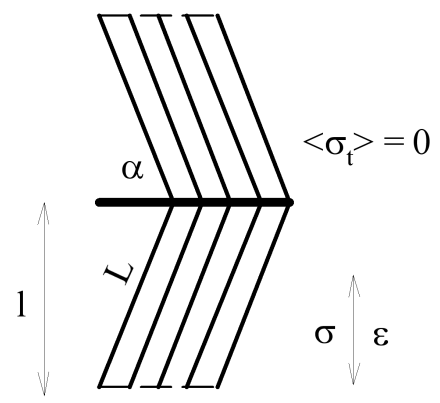

Fig. 4. Grain boundary barriers arising by in-plane tensile deformation.

Let us assume a grain pair in a simplified geometry after Fig. 4 where the traces of basal planes are indicated and the angle $\alpha$ is close to $90^{\circ}$. The increments of slip $\Delta \gamma$ and longitudinal strain $\Delta \varepsilon$ of grain pair are related to the change of $\alpha$ as follows:

$$
\Delta \gamma=\frac{\Delta \alpha}{\sin ^{2} \alpha}, \quad \Delta \varepsilon \equiv \frac{\Delta l}{l}=\cot \alpha \times \Delta \alpha .
$$

Some material remains, however, surrounding active grains that cannot accommodate deformation in the same plastic manner. Assuming an elastic accommodation in this case and considering a zero mean value of the transversal stress, $\left\langle\sigma_{\mathrm{t}}\right\rangle=0$, we can express the longitudinal accommodation stress by a Hook-like relation

$$
\Delta \sigma_{\mathrm{a}}=E^{*} \Delta \varepsilon,
$$

where the constant $E^{*} \leq E$ approximates the common Young modulus or may be less because of the possible effect of grain boundary sliding. The integration of 
Eqs. (1), (2) gives a maximum in the resolved accommodation stress $\tau_{\mathrm{a}}$ at an angle $\alpha_{\mathrm{m}}<90^{\circ}$. For a typical value of $\alpha \approx 82-83^{\circ}$ [1], it is $\alpha_{\mathrm{m}} \approx 85.7^{\circ}$ and the corresponding slip and strain $\gamma_{\mathrm{m}} \approx 0.056$ and $\varepsilon_{\mathrm{m}} \approx 0.0058$, respectively, when the latter value is comparable to the range in which hardening lifts appear. Thus, the linear expansion over the region from an initial angle $\alpha$ up to a maximum of $\alpha_{\mathrm{m}}$ yields

$$
\tau_{\mathrm{a}}=\frac{1+\nu}{2} \sin ^{2}(2 \alpha) \mu^{*} \gamma,
$$

where an accommodation shear modulus $\mu^{*} \leq \mu$ is introduced, $\nu$ is the Poisson ratio.

\subsection{The evolution of dislocation arrays}

The resistance contribution (3) is effectively connected with a boundary barrier $\hat{\tau}$ against the penetration of slip between the grains after Fig. 4. Assuming this barrier is overcome with the help of the pile-up of $N$ mobile dislocations over the length $L$, it holds [7]:

$$
\tau_{\mathrm{a}}^{2}=\frac{\mu b \hat{\tau}}{L}, \quad N \tau_{\mathrm{a}}=\hat{\tau},
$$

where $b$ is the Burgers vector amplitude. Defining now the density of pile-uped mobiles $\rho_{\mathrm{m}, \mathrm{a}}=N / L^{2}$, we obtain, with $(3)$, the relations

$$
\tau_{\mathrm{a}}=\mu b L \rho_{\mathrm{m}, \mathrm{a}}=C_{1} \frac{L}{b} \mu \gamma, \quad \rho_{\mathrm{m}, \mathrm{a}}=\frac{C_{1}}{b^{2}} \gamma,
$$

where the coefficient

$$
C_{1}=\frac{1+\nu}{2} \frac{b}{L} \frac{\mu^{*}}{\mu} \sin ^{2} 2 \alpha .
$$

For the usual multiplication mechanism, the increment of the mobile dislocation density governed by the area that these sweep, is given by a representative relation

$$
b^{2}\left(\frac{\mathrm{d} \rho_{\mathrm{m}}}{\mathrm{d} t}\right)_{\mathrm{gen}}=C_{\mathrm{g}} \frac{\mathrm{d} \gamma}{\mathrm{d} t}
$$

with an estimate $C_{\mathrm{g}} \approx 3 \times 10^{-5}$ (for fcc) reported in [8]. As to the above $C_{1}$, using $L=15 \mu \mathrm{m}, b=0.321 \mathrm{~nm}$, $\nu=0.26$, and $\alpha=82.5^{\circ}$, it is

$$
C_{1}=9 \times 10^{-7} \frac{\mu^{*}}{\mu} \ll C_{\mathrm{g}} .
$$

Consequently, the normal multiplication mechanism will safely supply the dislocations necessary to create the accommodation pile-ups.

According to (4), the density of primary mobiles, $\rho_{\mathrm{m}, 1}$, is strongly related to the primary slip. For the secondary mobiles, $\rho_{\mathrm{m}, 2}$, we accept the common evolution law [8] that involves (free) multiplication $\left(C_{1,2}\right)$, mutual trapping or dipole immobilization $\left(C_{2,2}\right)$, and the storage of mobiles governed by the principle of similitude (geometrical hardening, $C_{3,2}$ ). The network of stored dislocations of the density $\rho$ is assumed to be built-up by geometrical storage of both kinds of mobiles $\left(C_{3, i}\right)$ and to be reduced by the so-called glide (dynamic) recovery $\left(C_{4, i}\right)$. Thus, defining the reduced densities $y_{1}=b^{2} \rho_{\mathrm{m}, 1}, y_{2}=b^{2} \rho$, $y_{3}=b^{2} \rho_{\mathrm{m}, 2}$, we come to the following set of evolution equations:

$$
\begin{aligned}
& \dot{y}_{1}=C_{1} \dot{\gamma}_{1}, \\
& \dot{y}_{2}=\left(C_{3,1} \dot{\gamma}_{1}+C_{3,2} \dot{\gamma}_{2}\right) \sqrt{y_{2}}-\left(C_{4,1} \dot{\gamma}_{1}+C_{4,2} \dot{\gamma}_{2}\right) y_{2}, \\
& \dot{y}_{3}=\left(C_{1,2}-C_{2,2} y_{3}-C_{3,2} \sqrt{y_{2}}\right) \dot{\gamma}_{2},
\end{aligned}
$$

where $\dot{\gamma}_{1}, \dot{\gamma}_{2}$ are the primary and secondary slip rates, respectively.

\subsection{The flow stress}

Employing the well recognized square root law for the dislocation stress amplitude and the power law approach for the activation factor, we write the dislocation contribution to the flow stress as a product

$$
\begin{aligned}
\tau_{\mathrm{d} i} & =\left(\frac{\dot{\gamma}_{i}}{\Omega_{i} \nu_{i}}\right)^{\frac{1}{n_{i}}} \mu \alpha_{i} \sqrt{y_{2}}, \\
i & =1 \text { primary, } 2 \text { secondary, }
\end{aligned}
$$

where the parameters $\alpha_{i}$ of single interactions between the mobile and forest dislocations have been estimated to be about 1 [9], $\Omega$ is the elementary strain, $\nu_{0}$ is the attempt frequency, and the kinetic index $n$ is qualitatively given by the free energy $F_{0}$ of the elementary activation, $n \approx F_{0} / k T \gg 1$. For the primary regime of interest, the densities $\rho_{\mathrm{m}, 1}$ and $\rho$ vary slowly; therefore, we assume a constant factor $\dot{\omega}_{1}=\Omega_{1} \nu_{1}$. The secondary slip, however, begins at very low mobile densities $\rho_{\mathrm{m}, 2}$ and we therefore consider the exact dependence of the elementary strain on the densities (assuming a sole Burgers magnitude $b$ ): $\Omega_{2}=y_{3} / \sqrt{y_{2}}$.

The individual $i$-th slip mechanism can accommodate the imposed strain rate alone if the corresponding flow resistance at the full resolved rate $\dot{\varepsilon} / m_{i}$ remains less than the flow resistance for the remaining system at zero slip rate $\left(m_{i}\right.$ are the respective Schmid factors). The applied stress, $\sigma$, will then be balanced by the only active system: $m_{i} \sigma=\tau_{i}\left(\dot{\varepsilon} / m_{i}\right)$. If this single activity condition can be met for neither system, complex activity is established, and the glide rates of both the systems contribute to the applied rate

$$
\begin{gathered}
\frac{1}{m_{1}}\left[k_{1}+\mu \frac{L}{b} y_{1}+\tau_{\mathrm{d}, 1}\left(y_{1}, y_{2}, f\right)\right] \\
=\frac{1}{m_{2}}\left[k_{2}+\tau_{\mathrm{d}, 2}\left(y_{2}, y_{3}, f\right)\right]=\sigma .
\end{gathered}
$$

Here, the primary fraction $f=\frac{\dot{\gamma}_{1} m_{1}}{\dot{\varepsilon}}$ is introduced, the applied rate $\dot{\varepsilon}=m_{1} \dot{\gamma}_{1}+m_{2} \dot{\gamma}_{2}$, and the constants $k_{i}$ reflect the glide resistance components independent of the dislocation structures. We will perform the calculations under the identification of the primary as basal and the secondary as prismatic systems.

\subsection{The fit to experimental Mecking plots}

The necessary orientation parameters were drawn from the texture measurements taken before deformation and after tensile failure for both the $\mathrm{H} 24$ and $300 / 8$ structure 
states. Because the effects of state and deformation temperature seem to be minor, the mere anisotropy RD/TD is distinguished in Table. One can see that, as the strain increases, the ratio of the RD- and TD- orientation parameters "is inverted", this effect being extra marked for $\left\langle m_{1} / m_{2}\right\rangle$. The true positions of the hardening lifts on RD and TD tension tests may be achieved when the values $m_{2}$ and $m_{1} / m_{2}$ are chosen slightly after the "intersection point", especially $m_{2}=0.41$ and $0.40, m_{1} / m_{2}=0.801$ and 0.905 for RD and TD tension, respectively.

\section{TABLE}

Mean orientation parameters. $1=$ basal slip, $2=$ prismatic slip.

\begin{tabular}{c|c|c|c|c}
\hline \hline \multirow{2}{*}{} & \multicolumn{2}{|c|}{ Initial state } & \multicolumn{2}{c}{ After failure } \\
\cline { 2 - 5 } & $\left\langle m_{2}\right\rangle$ & $\left\langle m_{1} / m_{2}\right\rangle$ & $\left\langle m_{2}\right\rangle$ & $\left\langle m_{1} / m_{2}\right\rangle$ \\
\hline RD & 0.408 & 0.845 & 0.418 & 0.741 \\
TD & 0.424 & 0.717 & 0.370 & 1.235
\end{tabular}

The calculated Mecking plots for room temperature are shown in Fig. 5, in the caption of which the remaining model parameters are given. The values of attempt frequency $\nu_{2}$ and of the rate factor $\dot{\omega}_{1}$ seem to be rather small with respect to their reported estimates [7]. Yet, these discrepancies may be completely removed, assuming that only a minor part of the dislocation flow stress is subjected to thermal activation, whereas a dominant part of it remains practically athermal. Our preliminary relaxation experiments showed this athermal part to be about $85 \%$ (at $23{ }^{\circ} \mathrm{C}$ ) of the dislocation component and the kinetic exponent $n_{2} \approx 8.6$ was drawn. Then a quite satisfactory value $\nu_{2} \geq 10^{10} \mathrm{~s}^{-1}$ can be obtained. The value of the primary factor $\dot{\omega}_{1}$ could be commented in a similar way.

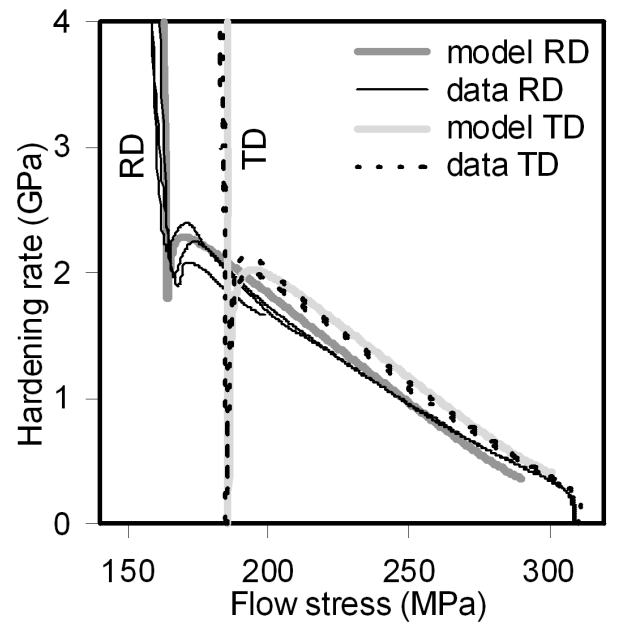

Fig. 5. Models for the Mecking plots, room temperature. The used parameters: $C_{1}=10^{-6}, L / b=40000$ $C_{1,2}=3.15 \times 10^{-4}, C_{2,2}=0.606, C_{3,1}=0.08, C_{3,2}=$ $0.077, C_{4,1}=9, C_{4,2}=15, k_{1}=40 \mathrm{MPa}, k_{2}=60 \mathrm{MPa}$ $\alpha_{1}=0.75, \alpha_{2}=1, \dot{\omega}_{1}=4.732 \times 10^{-3} \mathrm{~s}^{-1}, \nu_{2}=131 \mathrm{~s}^{-1}$, $n_{1}=10, n_{2}=20, \dot{\varepsilon}=1.33 \times 10^{-3} \mathrm{~s}^{-1}, \mu=16000 \mathrm{MPa}$, $y_{3 i}=1 \times 10^{-9}(\mathrm{TD}), 2 \times 10^{-8}(\mathrm{RD})$. For the values of $m_{2}$ and $m_{1} / m_{2}$ see text.
The striking difference in the drops of the hardening rate before the lifts for $\mathrm{RD}$ - and TD-regimes at room temperature can be modelled only assuming an anisotropy in the initial density of the secondary mobile dislocations $y_{3 i}$. In the case of TD, the deep drop corresponds to a minimum value reported for mobiles [8] $\rho_{\mathrm{m} 2, i} \approx 10^{10} \mathrm{~m}^{-2}$, whereas a 20 times higher density is connected to a less pronounced drop in the case of RD. At elevated temperature, however, the drops in hardening are comparable and shallow for either loading mode, Fig. 1b, which might be caused by an enhanced transformation of basal to prismatic dislocations by cross-slip.

\section{Conclusions}

- Based on the textures after deformation, double prismatic slip is proposed for the later tensile in-plane deformation.

- Accommodation within the rolled hexagonal structures induces a fast hardening of basal slip and consequently the transition to a secondary regime.

- Combining the evolution of dislocation populations with accommodation strengthening, the lifts in hardening rate, including their RD/TD anisotropy, are modelled.

\section{Acknowledgments}

This work was financed by the Ministry of Education of the Czech Republic under the Research Project 1M2560471601 Eco-centre for Applied Research of Non-Ferrous Metals. P.L. is grateful for the support offered by the Grant Agency of the Academy of Sciences of the Czech Republic under grant IAA201120902.

\section{References}

[1] S.R. Agnew, Ö. Duygulu, Int. J. Plasticity 21, 1161 (2005).

[2] J. Bohlen, M.R. Nürnberg, J.W. Senn, D. Letzig, A.R. Agnew, Acta Mater. 55, 2101 (2007).

[3] A. Jain, S.R. Agnew, Mater. Sci. Eng. A 462, 29 (2007).

[4] J. Balík, P. Lukáč, Z. Drozd, R. Kužel, Int. J. Mater. Res. 100, 322 (2009).

[5] J. Balík, P. Lukáč, J. Bohlen, K.U. Kainer, Kovove Mater. 45, 135 (2007).

[6] M.R. Barnett, Z. Keshavarz, X. Ma, Metall. Mater. Trans. A 37, 2283 (2006).

[7] U.F. Kocks, A.S. Argon, M.F. Ashby, Prog. Mater. Sci. 19, 1 (1975).

[8] L.P. Kubin, Y. Estrin, Acta Metall. Mater. 38, 697 (1990).

[9] B.M. Loginov, A.A. Predvoditelev, Phys. Status Solidi A 72, 69 (1982). 